\title{
Asma potencialmente fatal y disfunción de cuerdas vocales en paciente con estrés psicosocial como posible desencadenante de crisis asmáticas
}

\author{
Potentially fatal asthma and vocal chord dysfunction in \\ a patient with psychosocial stress as possible trigger for \\ asthma crises
}

\author{
Juan Sebastián Molina, MD ${ }^{(1)}$; AliRIo Rodrigo Bastidas, MD., MSc ${ }^{(2)}$; Amanda Doblado, TR ${ }^{(3)}$; \\ SANDRA TORO-HERRERA, MD ${ }^{(4)}$; LUIS FERnANDo GIRALDO, MD., PhD.(c) $)^{(5)}$
}

\begin{abstract}
Resumen
Los trastornos psiquiátricos son causas frecuentes de morbilidad en la población general, y su asociación con enfermedades orgánicas, entre ellas las patologías pulmonares, es frecuente. La relación entre las enfermedades mentales y dichas patologías puede desencadenar mala adherencia al tratamiento farmacológico, así como mayor uso de medicamentos controladores y aliviadores. El caso que se publica muestra la asociación entre asma potencialmente fatal con disfunción de cuerdas vocales, en donde el componente psicosocial personal podría desencadenar exacerbaciones severas con gran morbilidad para la paciente, deterioro en su calidad de vida y requerimiento de terapia farmacológica agresiva. Por esto es vital reconocer estos factores de estrés para iniciar una terapia integral, a fin de lograr mayor adherencia al tratamiento, disminuir las exacerbaciones y hospitalizaciones y evitar el escalonamiento terapéutico.
\end{abstract}

Palabras clave: asma, parálisis de cuerda vocal, trastornos mentales.

\begin{abstract}
Psychiatric disorders are a common causes of morbidity in general population and their association with organic diseases, including lung diseases is common. The relationship between mental illness and these diseases can lead to poor adherence to medical treatment, as well as increased use of controller medications and relievers. The case we present here shows the association between lung diseases, in this case near-fatal asthma with vocal cord dysfunction (VCD), where the personal psychosocial component could trigger severe exacerbations with significant morbidity for the patient, deteriorating her life quality and requiring aggressive drug therapy. This is why it is vitally to recognize these stressors in order to initiate an integral therapy, achieving greater adherence and decreased exacerbations, hospitalizations and avoiding escalation therapy.
\end{abstract}

Keywords: asthma, vocal cord paralysis, mental disorders.

\section{Introducción}

La prevalencia de enfermedades mentales asociadas a enfermedades respiratorias, es fuerte y existe una correlación bidireccional entre ambas ya que en los últimos años se ha adquirido mayor conocimiento en asociación con las posibles relaciones fisiopatológicas entre las enfermedades mentales y las orgánicas (1). En los pacientes con patologías respiratorias, puede diagnosticarse trastorno de ansiedad generalizado hasta en el $83 \%$, mientras que
${ }^{(1)}$ Médico Interno, Servicio de Neumología,
Clínica Universidad de La Sabana, Univer-
sidad de La Sabana. Chía, Cundinamarca,
Colombia.
(2)Internista, Neumólogo, Epidemiólogo Clí-
nico, Servicio de Neumología, Clínica Uni-
versidad de La Sabana. Chía, Cundinamarca,
Colombia.
(3)Terapeuta Respiratoria, Especialista en Pro-
moción en Salud y Desarrollo Humano, Clí-
nica Universidad de La Sabana. Chía, Cundi-
namarca, Colombia.
(4)Médico Psiquiatra, Servicio de Psiquiatría
Clínica Universidad de La Sabana. Chía,
Cundinamarca, Colombia.
(5)Internista, Neumólogo, PhD(c) Investigación
Clínica Aplicada. Director área de Investiga-
ción, Facultad de Medicina, Universidad de La
Sabana, Chía, Cundinamarca, Colombia.
Correspondencia: Juan Sebastián Molina.
Correo electrónico: jmolina1987@gmail.com
Recibido: 8-11-2013. Aceptado: 10-01-2014. 
el trastorno de pánico llega al $72 \%$ y el ataque de pánico al 73\% (2). Los pacientes asmáticos sufren trastornos de ansiedad y depresión hasta en el $61 \%$ (3); en la enfermedad pulmonar obstructiva crónica (EPOC) el trastorno depresivo mayor se ha asociado en el $40 \%$ (4) y hasta en el 15,8\% para el trastorno de ansiedad generalizada, prevalencia tres veces mayor cuando se compara con la población general (2).

En la disfunción de cuerda vocal (DCV) hay una asociación con alteraciones psiquiátricas hasta en el $73 \%$ y sus exacerbaciones son una causa frecuente de consulta a urgencias (5). El diagnóstico de la DCV en pacientes con asma y el control de las exacerbaciones asociadas a trastornos psiquiátricos, constituye un desafío para el tratamiento de estos pacientes (6). Si se logra el control adecuado de dichas comorbilidades se puede impactar la calidad de vida, ya que se ha determinado que aquellos pacientes con enfermedades pulmonares, particularmente asma y comorbilidades psiquiátricas asociadas, requieren mayor uso de medicamentos debido al pobre control de la enfermedad; por tanto al recibir un manejo integral de sus patologías psiquiátricas, hay mejores resultados en el control de los síntomas respiratorios (7).

Se expone el caso de una paciente con diagnóstico de asma y DCV, cuyas crisis eran desencadenadas, en su mayoría, por factores psicológicos personales, que requerían tratamiento intrahospitalario y escalonamiento farmacológico sin resultados satisfactorios.

\section{Caso clínico}

Paciente de sexo femenino, de 48 años de edad, ama de casa, quien ocho años atrás inició con un cuadro clínico de tos con expectoración blanquecina, sibilancias y compromiso severo de la función respiratoria; el cuadro cíclico, requirió soporte ventilatorio en varias ocasiones. Tenía, además, antecedente de rinitis, alergia a penicilina, cefalosporinas, trimetoprim/sulfametoxazol, dipirona y ácido acetil salicílico; no narró antecedentes de reflujo gastroesofágico.

Los ingresos a urgencias se caracterizaron, en su mayoría, por aparición de tos, asociado cronológicamente con estresores familiares o síntomas de miedo y desesperación, con aparición posterior de sibilancias y deterioro marcado del patrón ventilatorio, presentándose, en algunas ocasiones, alteración en el estado de conciencia y en la gasimetría arterial $\left(\mathrm{PCO}_{2}>50 \mathrm{~mm} \mathrm{Hg}\right)$, así como paro respiratorio. Esto tuvo impacto en su percepción de la enfermedad debido a que en el primer año de presentación de los síntomas aumentaban sus niveles de ansiedad y temor, ya que asociaba cualquier síntoma respiratorio a una crisis asmática, hecho que le impidió continuar ejerciendo su profesión debido a la frecuencia de exacerbaciones.

Fue tratada con múltiples esquemas de crisis y en siete ocasiones requirió ventilación mecánica invasiva con ingreso a la unidad de cuidados intensivos. El manejo crónico incluía terapia broncodilatadora permanente con salbutamol, bromuro de ipratropio, salmeterol/fluticasona, omalizumab durante seis meses y esteroide oral, sin mejoría clínica ni disminución en frecuencia de las exacerbaciones. La espirometría mostró un patrón obstructivo leve con respuesta significativa al broncodilatador (figura 1). La radiografía de tórax no evidenció alteraciones, y la tomografía computarizada de tórax fue normal.

Se realizó fibrobroncoscopia, la cual no evidenció lesiones obstructivas en árbol bronquial y nasofibrolaringoscopia basal sin alteraciones anatómicas; posterior al ejercicio hasta alcanzar frecuencia cardiaca máxima, se observaron cuerdas vocales falsas edematizadas, con adecuado cierre al estímulo; con oxígeno a 2 y 5 litros las cuerdas vocales tuvieron cierre parcial, dejando orificio posterior triangular con buena motilidad de la cuerda vocal derecha y disminución de la motilidad de la izquierda. El aspecto de la cuerda vocal derecha fue normal, y la izquierda mostró pérdida de la refringencia; sin embargo, hubo cierre parcial al inducir los movimientos con la fonación, y se hizo diagnóstico de disfunción de cuerdas vocales (figura 2).

Durante su última hospitalización requirió intubación orotraqueal con ventilación mecánica prolongada, traqueostomía y oxígeno domiciliario adicional al manejo broncodilatador. Fue valorada por el servicio de Psiquiatría que consideró que la 


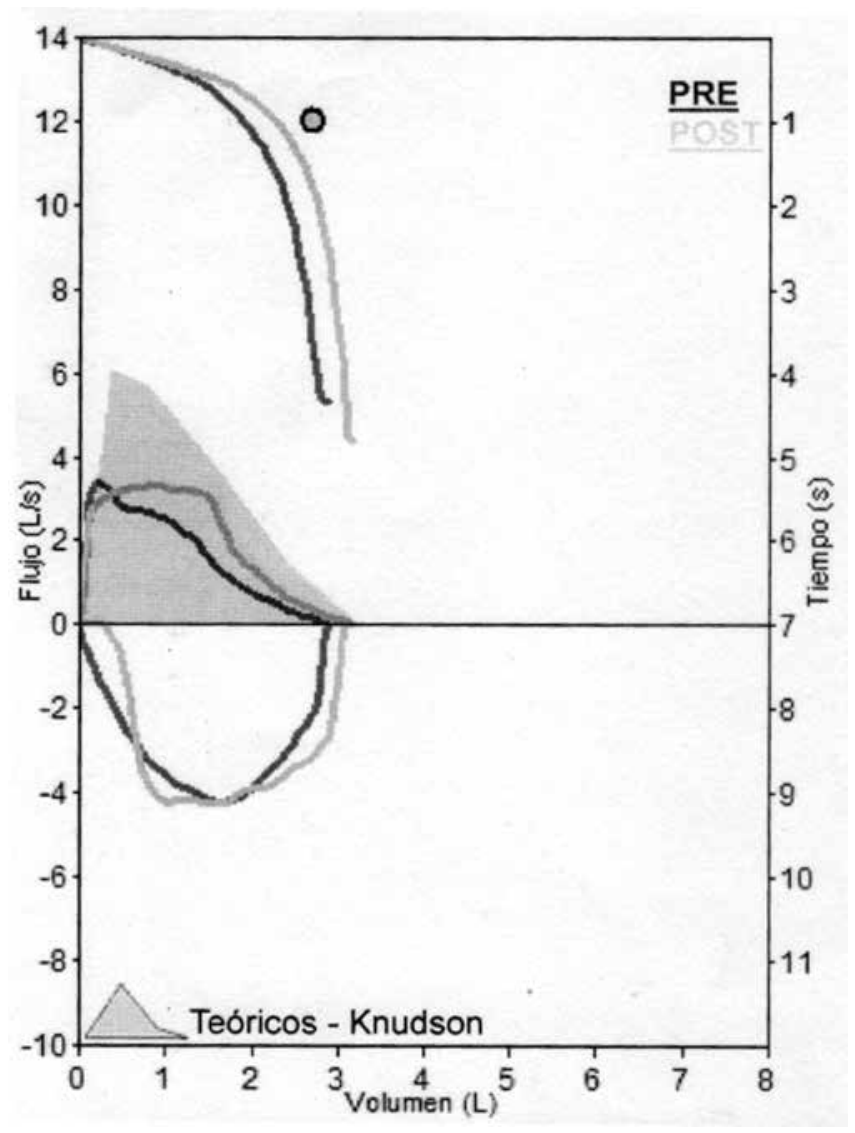

\begin{tabular}{|l|c|c|}
\hline \multicolumn{1}{|c|}{ PARÁMETROS } & Pre B2 & Post B2 \\
\hline FVC & 2,89 & 3,16 \\
\hline FEV1 & 1,97 & 2,30 \\
\hline FEV1\% & 73 & 85 \\
\hline FEF $25-75$ & 1,46 & 1,90 \\
\hline
\end{tabular}

Figura 1. Curva flujo/volumen pre y post broncodilatador.

paciente tenía rasgos de personalidad obsesivos, sin observarse ganancia secundaria en lo referente a la presencia de síntomas.

\section{Discusión}

La DCV, también llamada movimiento paradójico de cuerdas vocales, se caracteriza por un movimiento anormal uni o bilateral de las cuerdas vocales durante la inspiración en ausencia de una patología u origen orgánico (8), siendo más frecuente en el género femenino (3:1) (9). Los datos estadísticos son

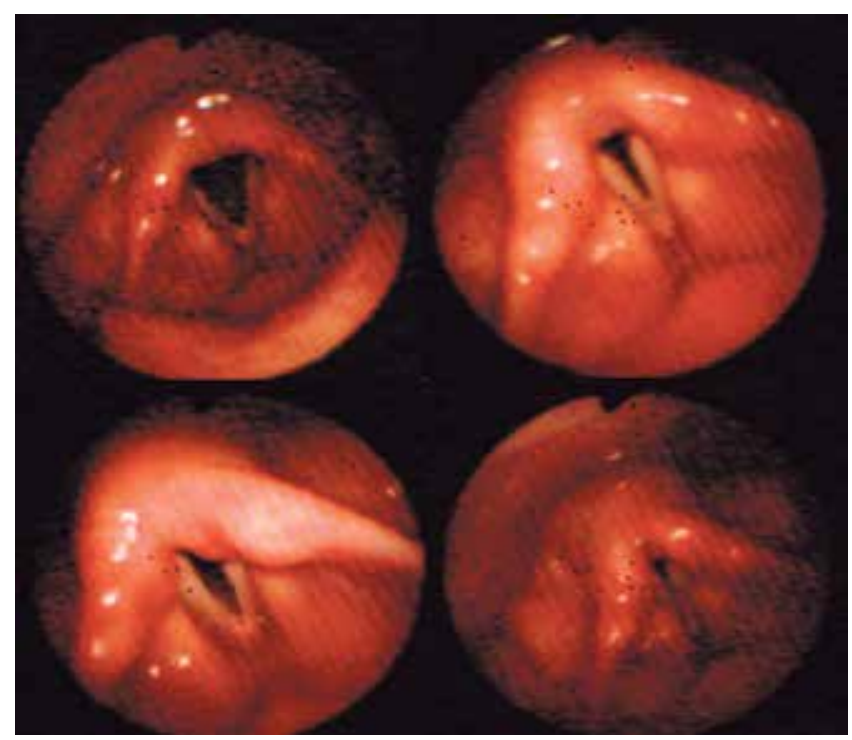

Figura 2. Nasofibrolaringoscopia basal y post ejercicio que permitió el diagnóstico de disfunción de las cuerdas vocales.

pobres debido a la falta de estudios en grandes poblaciones, por lo que se ha estudiado únicamente en grupos específicos, como el caso de pacientes con asma (8). En adultos con asma refractaria, hasta el $10 \%$ pueden padecer DCV y cerca del $22 \%$ de las consultas recurrentes a los servicios de urgencias se asocia con episodios súbitos de disnea relacionada con laringoespasmo (8).

En el caso expuesto la alteración obstructiva en la espirometría, la disfunción en la movilidad de las cuerdas vocales evidenciada en la nasofibrolaringoscopia, en conjunto con la evaluación psiquiátrica, conformaron un diagnóstico de asma asociado a DCV y rasgos de personalidad dentro del espectro ansioso.

Según Sears y colaboradores los episodios severos en la enfermedad asmática con paro respiratorio, la elevación de la presión arterial de $\mathrm{CO}_{2}$ y/o la alteración del estado de consciencia definen el cuadro conocido como asma potencialmente fatal (10). En esta entidad se describen dos fenotipos $(11,12)$ : el tipo 1, que equivale al $80 \%-90 \%$ de los casos en los que el factor desencadenante son las infecciones respiratorias con una respuesta terapéutica lenta, y el tipo 2 (características presentes en la paciente del caso), donde los adultos tiene una evolución rápida 
con asfixia, y cuyos desencadenantes son estresores psicosociales así como alergenos respiratorios (12).

La DCV, por otra parte, se asocia con condiciones orgánicas y cerca del $40 \%$ al $73 \%$ con condiciones psicosomáticas como ansiedad y depresión (8, 13-15). Lavoie y colaboradores. reportaron que aquellos pacientes con comorbilidades psiquiátricas muestran peor control de su patología pulmonar, independiente de la edad, el género y hábito tabáquico, así como el mayor uso de broncodilatadores de corta acción (9).

Dentro de las explicaciones del porqué los episodios agudos de las enfermedades mentales se relacionan con cuadro de exacerbación en patologías pulmonares como en el asma, se describe el "hiperalertamiento", un componente psicogénico que puede estar presente en dicha enfermedad. Lamandescu sugiere también que la hipocapnia secundaria a la hiperventilación, común en estados agudos de ansiedad, puede desencadenar espasmos bronquiales y generar así crisis asmáticas en pacientes con cierta susceptibilidad genética (8). Por otro lado, la alcalosis respiratoria, que lleva a una alteración en el manejo del calcio intracelular en sujetos con alteraciones en la reparación celular (vía de la menor resistencia, Lindeman y colaboradores) puede llevar a hipertensión pulmonar, y conllevar mayor hipoxemia y disnea (16).

De otro lado, los trastornos psiquiátricos y las exacerbaciones respiratorias pueden estar asociados con la producción de ciertos neurotransmisores, fenómeno que conduce a una "incoordinación" en el funcionamiento normal del aparato vocal de los sujetos con DCV (17). Los individuos con alteraciones mentales sobreproducen acetilcolina y otras sustancias como la sustancia P, neurocininas A y B y péptido relacionado con el gen de calcitonina los cuales estimulan el nervio vago a través del sistema autónomo parasimpático, y pueden llevar a broncoespasmo sin predominio de la hipersecreción bronquial. Las situaciones de estrés crónico o ansiedad prolongada, también se relacionan con disminución en la producción de cortisol, que progresa a una disregulación de los procesos anti y pro inflamatorios, generando mayor susceptibilidad a desencadenar respuestas broncoconstrictoras (17).
En el tratamiento es esencial tener en cuenta un abordaje multidisciplinario. El tratamiento recomendado para el asma es el broncodilatador; en cuanto a la DCV son útiles la psicoterapia conductual (18) y los ejercicios de respiración con la utilización de dispositivos como el threshold inspiratorio, el cual al aumentar la eficiencia de los músculos de la vía aérea superior durante la inspiración, relaja las cuerdas vocales, eliminando el estridor (19). También se ha utilizado la toxina botulínica que inhibe la liberación de acetil colina y relaja las cuerdas vocales (20), evitando así el trauma de procesos como la traqueostomía (reservados para casos severos); sin embargo, esta opción puede producir disfagia y aspiración.

El tratamiento farmacológico de las enfermedades mentales es una de las medidas más importantes para el control de exacerbaciones de las enfermedades respiratorias en estos pacientes. En este aspecto, los inhibidores selectivos de recaptación de serotonina son los más estudiados y han demostrado un mejor perfil de seguridad y así como menor requerimiento de corticosteroides sistémicos a largo plazo $(21,22)$. Aunque en la paciente del caso no se ha documentado un trastorno definido, teniendo en cuenta lo observado durante la atención en urgencias y hospitalización, y que su personalidad tiene rasgos del grupo $\mathrm{C}$, es válido hacer un seguimiento que permita dilucidar si hay un trastorno de ansiedad de base o un control pobre de las respuestas a la ansiedad que generen mal control y exacerbación de los síntomas respiratorios.

\section{Conclusión}

Además de mejorar la calidad de vida de los pacientes, el control de las enfermedades mentales puede influir en la regulación de la liberación de los neurotransmisores hasta ahora implicados en el control pobre y la exacerbación de las enfermedades respiratorias; asimismo, el manejo de la patología respiratoria con broncodilatadores puede ser beneficioso en algunos pacientes con DCV y están indicados para el manejo de las exacerbaciones y el control de la enfermedad de base. En este tipo de patologías se propone realizar un seguimiento a cargo del servicio de Psiquiatría, en conjunto con una entrevista familiar, un registro del pensamiento y la 
implementación de técnicas de relajación y de distracción del pensamiento, lo que puede brindar alternativas que pueden mejorar la calidad de vida del paciente y su familia.

Los trastornos mentales deben considerarse en pacientes con cuadros severos y recurrentes de DCV y asma. El manejo de las mismas puede ayudar a controlar y mejorar la calidad de vida de estos pacientes, prescribir menos medicamentos de larga acción y disminuir a largo plazo los ingresos hospitalarios y las consultas a servicios de urgencias.

\section{Bibliografía}

1. Jain A, Lolak S. Psychiatric aspects of chronic lung disease. Current psychiatry Reports. 2009;11:219-25.

2. Shanmugam G, Bhutani S, Khan DA, Brown ES. Psychiatric considerations in pulmonary disease. Psychiatric Clin North Am. 2007;30:76180

3. Brenes GA. Anxiety and chronic obstructive pulmonary disease: prevalence, impact, and treatment. Psychosomatic Medicine. 2003;65:96370.

4. Balkinssoon R, Norwood R. Current perspectives on management of co-morbid depression in COPD. COPD. 2005;2:185-93.

5. Jain S, Bandi V, Zimmerman J, Hanania N, Guntupalli K. Incidence of vocal cord dysfunction in patients presenting to emergency room with acute asthma exacerbation. CHEST. 1999;11:243.

6. Benninger C, Parsons JP, Mastronarde JG. Vocal cord dysfunction and asthma. Current Opinion in Pulmonary Medicine. 2011;17:45-9.

7. Lavoie KL, Cartier A, Labrecque M, Bacon SL, Lemière C, Malo J-L, et al. Are psychiatric disorders associated with worse asthma control and quality of life in asthma patients. Respir Med. 2005;99:1249-57.

8. Truong A, Truong DT. Vocal cord dysfunction: an updated review.
Otolaryngol. 2011;2:2.

9. Mobeireek A, Alhamad A, Al-Subaei A, Alzeer A. Psychogenic vocal cord dysfunction simulating bronchial asthma. Eur Resp Jl. 1995;8(11):1978-81

10. Molfino NA, Slutsky AS. Near-fatal asthma. Eur Respir J. 1994;7:98190

11. Restrepo RD, Peters J. Near-fatal asthma: recognition and management. Curr Opin Pulm Med. 2008;14:13-23.

12. Rodrigo GJ. Asma potencialmente fatal o de riesgo vital. [Consultado Mar 25 de 2013]; Disponible en: http://www.ramr.org.ar/archivos/numero/ano_9_4_dic_2009/mere4_1.pdf

13. Morris M, Christopher K. Diagnostic criteria for the classification of vocal cord dysfunction. CHEST. 2012;138:1213-23.

14. Munro S. Diagnosis and management of vocal cord dysfunction. J Nurse Practitioners. 2010;6:675-82.

15. Morris M. Vocal cord dysfunction: etiologies and treatment. Clin Pulm Med. 2006;13:73-86.

16. Kalinin V. Anxiety disorders. 1st. ed. Rijeka, Croatia: InTech; 2011.

17. Lamandescu I. Involvement of psychological stimuli in the pathogenic context of bronchial asthma. Info Medica. 2009;2:148.

18. Thurston N, Fiedorowicz J. Improvement of paradoxical vocal cord dysfunction with integrated psychiatric care. Psycosomatics. 2009;50:2824.

19. Weiner P, Azgad Y, Ganam R, Weiner M. Inspiratory muscle training in patients with bronchial asthma. CHEST. 1992;102:1357-61.

20. Maillard I, Schweizer V, Broccard A, Duscher A. Use of botulinum toxin type A to avoid tracheal intubation or tracheostomy in severe paradoxical vocal cord movement. CHEST. 2000;118:874-6.

21. Sherwood E, Howard C, Khan DA, Carmody T. Escitalopram for severe asthma and major depressive disorder: a randomized, doubleblind, placebo-controlled proof-of-concept study. Psycosomatics. 2012;53:75-80.

22. Brown ES, Vigil L, Khan DA, Liggin JDM, Carmody TJ, Rush AJ. A randomized trial of citalopram versus placebo in outpatients with asthma and major depressive disorder: a proof of concept study. Biological Psychiatry. 2005;58:865-70. 